\title{
The role of waveform monitoring in Sepsis identification within the first hour of Intensive Care Unit stay*
}

\author{
Maximiliano Mollura ${ }^{1}$, Giulio Mantoan ${ }^{1}$, Stefano Romano ${ }^{1}$, Li-wei Lehman ${ }^{2}$ Senior Member, IEEE, \\ Roger G. Mark ${ }^{2}$ Fellow, IEEE and Riccardo Barbieri ${ }^{1}$ Senior Member, IEEE
}

\begin{abstract}
The latest extensive development of machine learning models in healthcare, and in particular their application to data from the intensive care unit (ICU), is directed towards the main objective to help clinicians in making more timely diagnoses and efficient decisions. Many studies have been focused on the identification of Sepsis in a complex environment such as the ICU by using the data collected in electronic health records. However, only a few studies have investigated associations between the patients' continuously monitored vital signs and their Sepsis status.

This work aims at demonstrating that machine learning algorithms considering measures extracted from 103 patients from the publicly available MIMIC-III clinical and waveform databases are able to adequately identify Sepsis just within the first hour of stay in the ICU.

A bagged tree classifier showed $\mathrm{AUC}=0.86$, Specificity $=0.85$ and Sensitivity $=0.86$ on the test set, when trained using only the information extracted from the recorded electrocardiogram and arterial blood pressure waveforms, showing that the information coming from waveform monitoring may help in detecting Sepsis within the first hour of ICU stay.
\end{abstract}

\section{INTRODUCTION}

Patients staying in the Intensive care unit (ICU) are characterized by unstable conditions due to the presence of a wide range of pathologies, injuries, or post-surgery traumas. According to the third international consensus definitions for Sepsis and Septic Shock [1], one of the major causes of mortality in ICU is represented by Sepsis and its reported incidence is increasing. To this extent, the development of algorithms able to identify this infectious state is of primary importance.

Patients entering the ICU are constantly monitored by nurses, clinicians and medical instrumentation; the availability of electronic health records (EHR) and continuously recorded vital signs such as the electrocardiogram (ECG) and arterial blood pressure (ABP) waveforms, all containing useful information regarding the patient's health state, have allowed for the development of algorithms that predicted Sepsis up to 4 hours prior to its onset. [2][3]

Because the definition of a Sepsis onset is affected by significant uncertainties mainly due to the difficulties in associating clinical measures to a precise onset time, this

\footnotetext{
*This work was partially supported by Progetto Roberto Rocca, MIT-Italy Program

1 Maximiliano Mollura, Giulio Mantoan, Stefano Romano and Riccardo Barbieri are from the Department of Electronic, Information and Biomedical Engineering, Politecnico di Milano. maximiliano.mollura@polimi.it

2 Li-wei Lehman and Roger G. Mark are from the Laboratory for Computational Physiology, Massachusetts Institute of Technology
}

work aims at identifying septic subjects, at any stage of development of the infection, within the first hour of the patients' stay. The identification is performed by a machine learning classifier fed by heartbeat dynamics and cardiovascular features extracted from the continuously recorded ECG and ABP signals.

\section{MATERIALS AND METHODS}

\section{A. Cohort and Data Selection}

Data used in this study were extracted from the MIMICIII database [4], containing EHR and continuously recorded physiological signals from patients admitted between 2001 and 2012 in the Beth Israel Deaconess Medical Center (BIDMC, Boston, Massachusetts) ICU.

As it is advisable to identify septic subjects as soon as possible after their admission in the ICU, we extracted a reliable set of measures of the patients' health state within the first 1-hour window from admission. The availability of 1hour recordings in the first hour of intensive care was defined according to the following characteristics:

- Age higher than 18 years old at the admission in ICU.

- Presence of clinical information.

- Data recorded by MetaVision system (2008-2012)

- Presence of both ECG, labeled as either I, II or "V" lead, and ABP recordings.

- Less than $50 \%$ of missing values within each 1-hour recording window.

- Less than $50 \%$ of noise such as saturation, triggers, motion or sensor calibration loss.

According to the selection criteria above, a total of 103 subjects were labeled as 'septic' or 'control' whether they did or did not develop Sepsis at any time during their hospital stay, according to the third definition of Sepsis [1], resulting in 39 septic $(\mathrm{S})$ patients and 64 controls $(\mathrm{C})$.

Demographics (percentage of female subjects, median and interquartile ranges for age and length of stay (LOS), percentages of in-hospital (Hosp.) and within 90-day (90 days) from discharge mortality) and percentages of co-morbidities (congestive heart failure (CHF), diabetes (Diab.), renal failure (RF), liver disease (LD) and coagulopathy (CGPT)) for each of the two groups are shown in Table I.

A set of features from the ECG and ABP waveforms recorded from each subject was extracted. We annotated the R-peak positions from the ECG, as well as the systolic and diastolic positions and values from the ABP. Through the systolic (SAP) and diastolic (DAP) time series we extracted the 
TABLE I

DEMOGRAPHICS AND PERCENTAGES OF CO-MORBIDITIES OF THE CONSIDERED COHORT ( $\mathrm{C}=$ CONTROL, $\mathrm{S}=$ SEPTIC GROUPS $)$.

\begin{tabular}{|l||l|l|l|l|l|}
\hline \multicolumn{6}{|c|}{ Demographics } \\
\hline & Gender (\%F) & Age (yr) & LOS (days) & Hosp. & 90 days \\
\hline C & 0.67 & $52(45-66)$ & $1.1(0.9-2)$ & 0.01 & 0.04 \\
S & 0.39 & $57(45-65)$ & $2(1.1-4)$ & 0.02 & 0.05 \\
\hline \hline \multicolumn{5}{|c|}{ Co-morbidities } \\
\hline & CHF & Diab. & RF & LD & CGPT \\
\hline C & 0 & 0.09 & 0.06 & 0.08 & 0.04 \\
S & 0.04 & 0.08 & 0.03 & 0.08 & 0.03 \\
\hline
\end{tabular}

mean arterial pressure (MAP) and the pulse arterial pressure (PAP) as the pointwise difference between SAP and DAP. From the RR series we extracted the heart rate variability (HRV) linear (AVNN, SDNN, SDANN, etc...), non-linear (Entropy and Lyapunov, DFA and Hurst exponents) and spectral domain (VLF, LF, HF, LFn, HFn powers and LF/HF) measures. In addition, a point-process modeling of the RR series [5] allowed for the extraction of high resolution timevarying spectral domain measures, thus allowing to compute their first four statistical moments, first, second and third quartiles and the coefficient of the linear regression in time within the recording hour. Similar measures are computed for SAP, DAP, MAP and PAP.

\section{B. Feature Selection and Classification}

The set of observations was divided into training and test sets with a stratified hold-out $80 \%-20 \%$ partition. Then, a feature selection step was performed with LASSO.

Logistic regression (LR), Discriminant Analysis (DA), Trees, Support Vector Machines (SVM), Bagged Trees (B.TREE) and Random Undersampling Boosting (RUS) classifiers were trained in a leave-one-out cross-validation in order to optimize their hyperparameters with a Bayesian optimization rule. The selected features were: Hurst exponent, slope of the linear regression of the dynamic sympatho-vagal balance, kurtosis of the mean arterial pressure, Lyapunov exponent, sample entropy, kurtosis of the pulse pressure, TINN, first quartile and standard deviation of DAP and the slope of the linear interpolation of the RR spectrum in a log$\log$ scale. The trained models were tested on the test set. Area under the receiving operating characteristic curve (AUC), Sensitivity (SE), Specificity (SP), Accuracy (ACC), F1 score (F1), Positive Predictive Value (PPV) and Negative Predictive Value (NPV) were considered as performance measures. All analyses were performed with MATLAB 2019a.

\section{RESULTS}

Among all the classifiers, the RUS showed the highest AUC $=0.92$ with good results also on NPV (0.83), SE (0.71), SP (0.77) and ACC (0.75). A bagged tree model obtained the highest $\mathrm{F} 1=0.8, \mathrm{PPV}=0.75, \mathrm{NPV}=0.92, \mathrm{SE}=0.86, \mathrm{SP}=0.85$ and $\mathrm{ACC}=0.85$ scores and the second best $\mathrm{AUC}=0.86$.

The obtained performance measures are shown in Table II. The presented results are comparable with classification scores obtained with NEWS ( $\mathrm{SE}=0.84, \mathrm{SP}=0.85$, $\mathrm{AUC}=0.91), \quad \mathrm{SIRS} \quad(\mathrm{SE}=0.86, \quad \mathrm{SP}=0.79, \quad \mathrm{AUC}=0.88)$ and
TABLE II

RESUlTS OBTAINED IN SEPSIS IDENTIFICATION ON THE TEST SET WITH DIFFERENT CLASSIFICATION ALGORITHMS.

\begin{tabular}{|c||c|c|c|c|c|c|}
\hline \multicolumn{7}{|c|}{ Identification Results } \\
\hline & TREE & B.TREE & SVM & LR & DA & RUS \\
\hline AUC & 0.74 & 0.86 & 0.73 & 0.8 & 0.79 & $\mathbf{0 . 9 2}$ \\
F1 & 0.67 & $\mathbf{0 . 8}$ & 0.36 & 0.59 & 0.53 & 0.67 \\
PPV & 0.63 & $\mathbf{0 . 7 5}$ & 0.5 & 0.5 & 0.5 & 0.63 \\
NPV & 0.83 & $\mathbf{0 . 9 2}$ & 0.69 & 0.8 & 0.75 & 0.83 \\
SE & 0.71 & $\mathbf{0 . 8 6}$ & 0.29 & 0.71 & 0.57 & 0.71 \\
SP & 0.77 & $\mathbf{0 . 8 5}$ & $\mathbf{0 . 8 5}$ & 0.62 & 0.69 & 0.77 \\
ACC & 0.75 & $\mathbf{0 . 8 5}$ & 0.65 & 0.65 & 0.65 & 0.75 \\
\hline
\end{tabular}

qSOFA $(\mathrm{SE}=0.29, \mathrm{SP}=0.989, \mathrm{AUC}=0.81)$ scores at the emergency department [6]. Detection time (60 minutes recording) is comparable to SIRS and qSOFA documentation times from arrival in the emergency department (47.1 and 84 mins, respectively). [7]

\section{CONCLUSIONS}

Our results suggest that in the first hour of ICU stay, when the information coming from the laboratory measures is not completely available, measures extracted from the recorded patients' vital sign waveforms might help in identifying Sepsis and the obtained results are also comparable with those obtained with NEWS, SIRS and qSOFA scores.

Further studies will focus on consideration of a larger patient cohort as well as a more refined investigation of the ability of the model in discriminate Sepsis from hemorrhage, cardiogenic shock and dehydration.

In conclusion, results suggest that a satisfactory septic status identification might be obtained using data recorded in the first hour of ICU stay, thus potentially avoiding the need of an explicit definition of Sepsis onset independently from the stage of development of the infection.

\section{REFERENCES}

[1] M. Singer, C. S. Deutschman, C. W. Seymour et al. The Third International Consensus Definitions for Sepsis and Septic Shock (Sepsis-3). JAMA. 2016;315(8):801-810. doi:10.1001/jama.2016.0287.

[2] S.P. Shashikumar, M.D. Stanley, I. Sadiq, Q. Li, A. Holder, G.D. Clifford, and S. Nemati. Early sepsis detection in critical care patients using multiscale blood pressure and heart rate dynamics. Journal of Electrocardiology, Volume 50, Issue 6, 2017, Pages 739-743, ISSN 0022-0736, https://doi.org/10.1016/j.jelectrocard.2017.08.013.

[3] S. Nemati, A. Holder, F. Razmi, M.D. Stanley, G.D. Clifford, and T.G. Buchman. An interpretable machine learning model for accurate prediction of sepsis in the icu. Critical care medicine, 46(4), 547-553. doi:10.1097/CCM.0000000000002936

[4] A. E. W. Johnson , T.J. Pollard L. Shen, L. Lehman, M. Feng, M. Ghassemi, B. Moody, P. Szolovits, L.A. Celi and R.G. and Mark. MIMIC-III, a freely accessible critical care database.Scientific data, 3, 160035. doi:10.1038/sdata.2016.35.

[5] R. Barbieri \& E. Matten \& A. Alabi, \& E. Brown. (2005). A pointprocess model of human heartbeat intervals: New definitions of heart rate and heart rate variability. Am J Physiol Heart Circ Physiol. 2005 Jan; 288(1):H424-35. doi: 10.1152/ajpheart.00482.2003.

[6] O.A. Usman, A.A. Usman, M.A. Ward, Comparison of SIRS, qSOFA, and NEWS for the early identification of sepsis in the Emergency Department, The American Journal of Emergency Medicine, Volume 37, Issue 8, 2019, Pages 1490-1497, ISSN 0735-6757. doi: 10.1016/j.ajem.2018.10.058.

[7] S. Haydar, M. Spanier, P. Weems, S. Wood, T. Strout. Comparison of QSOFA score and SIRS criteria as screening mechanisms for emergency department sepsis, The American Journal of Emergency Medicine, Volume 35, Issue 11, 2017, Pages 1730-1733, ISSN 07356757. doi: 10.1016/j.ajem.2017.07.001. 